\title{
Interesting species of longhorn beetles (Coleoptera: Cerambycidae) from China in the collection of S. Murzin. Part 1
}

\section{Интересные виды жкуков-усачей (Coleoptera: Cerambycidae) из Китая в комлекщии С. Мурзина (Coleoptera: Cerambycidae). Часть 1}

\author{
M.A. Lazarev*, S.V. Murzin**

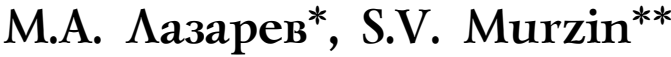

\begin{abstract}
* Free Economic Society of Russia, Department of Scientifics Conferences and All-Russian Projects, Tverskaya Str. 22a, Moscow 125009 Russia. E-mail: cerambycidae@bk.ru; humanityspace@gmail.com.

* Вольное экономическое общество России, Департамент научных конференций и Всероссийских проектов, Тверская ул. 22a, Москва 125009 Россия.

** Proletarsky prosp. 8, build. 1, apart. 23, Moscow 115522 Russia. E-mail: murka3@list.ru.

** Пролетарский просп. д. 8, корп. 1, кв. 23, Москва 115522 Россия.
\end{abstract}

Ключевые слова: Coleoptera, Cerambycidae, новые находки, Китай.

Key words: Coleoptera, Cerambycidae, new records, China.

Abstract. 20 Cerambycidae species are newly recorded for different provinces of China, and three species, Annamanum rondoni Breuning, 1962, Rhodopina albomarmorata Breuning, 1958 and $R$. pedongensis Breuning, 1969, are recorded from China for the first time.

Резюме. В статье приводится информация о 20 видах жуков-усачей, которые впервые указываются для различных провинций Китая, а Annamanum rondoni Breuning, 1962, Rhodopina albomarmorata Breuning, 1958 и Rhodopina pedongensis Breuning, 1969 впервые указывается для Китая.

\section{Introduction}

Many new interesting Cerambycidae were collected all over the world by Sergey Murzin during several seasons and preserved in a private home collection, which was never systematically observed. Now we begin to study that enormous materials. The results of our investigations will be published in a series of articles.

The identification of the species were arranged by the authors. Sometimes it was kindly proved by Dr. Meiying Lin, Dr. Guanglin Xie and Dr. Junsuke Yamasako.

The distributional data for each taxon are based on the publication by Lin and Yang [2019].

All materials studied are deposited in the collection of S. Murzin (Moscow, Russia).

\section{List of identified species}

$$
\begin{gathered}
\text { Acalolepta (Acalolepta) seunghwani } \\
\text { Danilevsky, } 2013
\end{gathered}
$$

Material. China: Sicbuan prov., $70 \mathrm{~km}$ W Chengdu, Qingcheng Hou Shan mts, $1500 \mathrm{~m}, 8-14.06 .2005$, S. and V. Murzin leg. -1 male; $70 \mathrm{~km} \mathrm{~W} \mathrm{Chengdu,} \mathrm{Qingcheng} \mathrm{Hou}$ Shan mts, $1500 \mathrm{~m}, 15-20.06 .2005$, S. and V. Murzin leg. -
1 female, (SM); Oingchenghoushan Mts, $70 \mathrm{~km} \mathrm{~W} \mathrm{Chengdu,}$ $1435 \mathrm{~m}$, 9-14.07.2004, S. Murzin leg. - 1 male; Qingchenghoushan Mts, $1400 \mathrm{~m}, 16-25.08 .2005$, S. Murzin leg. - 1 male; Qingchenghoushan Mts, 1400 m, 16-25.08.2005, S. Murzin leg. - 1 male; Qingchenghoushan Mts, $1400 \mathrm{~m}$, 21-25.06.2005, S. Murzin leg. - 1 male; Oingchenghou Shan Mts, $70 \mathrm{~km} \mathrm{NW} \mathrm{Chengdu,} 1450 \mathrm{~m}$, 10.07.2004, S. Murzin leg. - 1 female; Qingchenghou Mts, $70 \mathrm{~km} \mathrm{~W} \mathrm{Chengdu,}$ $1500 \mathrm{~m}$, 15-18.07.2004, S. Murzin leg. - 1 male; Qingchenghou Mts, $70 \mathrm{~km} \mathrm{~W} \mathrm{Chengdu,} 1500 \mathrm{~m}, 15-$ 18.07.2004, S. Murzin leg. - 1 male; Qingchenghou Shan Mts, $70 \mathrm{~km}$ NW Chengdu, $1450 \mathrm{~m}, 15.08 .2004$, S. Murzin leg. 1 male; Qingcheng Houshan Mts, $70 \mathrm{~km} \mathrm{NW}$ Chengdu, 30.9338 N, 103.4738 E, 1350 m, 6-13.08.2010, S. Murzin leg. -1 male.

Distribution. China (Sichuan — new record; Jilin), DPR Korea, RO Korea, Russia (Siberia).

Acalolepta (Acalolepta) vitalisi (Pic, 1925)

Material. China: W Yunnan prov., $60 \mathrm{~km}$ E Tengchong mts, $2200 \mathrm{~m}, 19-22.05 .2006$, S. Murzin and I. Shokhin leg. 1 female.

Distribution. China (Yunnan - new record; Zhejiang, Jiangxi, Hunan, Fujian, Taiwan, Guangdong, Hainan, Guangxi, Sichuan), Vietnam, Cambodia.

\section{Paraleprodera carolina (Fairmaire, 1900)}

Material. China: $S$ Gansu prov., left bank of Yantanghe, $33^{\circ} 13^{\prime} 35^{\prime \prime} \mathrm{N}, 104^{\circ} 42^{\prime} 29^{\prime \prime} \mathrm{E}, 1673$ m, 19.07.2004, I. Belousov, I. Kabak leg. -1 female.

Distribution. China (Gansu - new record; Shaanxi, Jiangsu, Zhejiang, Hubei, Jiangxi, Hunan, Fujian, Taiwan, Sichuan, Guizhou, Yunnan).

\section{Pharsalia (Antennopharsalia) antennata Gahan, 1894}

Material. China, Sichuan prov., Oingchenghou Shan Mts, $70 \mathrm{~km} \mathrm{NW}$ Chengdu, $1450 \mathrm{~m}, 15.08 .2004$, S. Murzin leg. -1 male.

Distribution. China (Sichuan - new record; Fujian, Guangxi, Yunnan), Laos, India, Myanmar. 


\section{Xenohammus bimaculatus Schwarzer, 1931}

Material. Cbina: Sicbuan, $70 \mathrm{~km} W$ Chengdu, Qingcheng Hou Shan mts, $1500 \mathrm{~m}, 15-20.06 .2005$, S. and V. Murzin leg. - 1 male; Qingchenghou Shan Mts, $70 \mathrm{~km} \mathrm{NW} \mathrm{Chengdu,}$ $1450 \mathrm{~m}, 15.08 .2004$, S. Murzin leg. -2 females; Baoxing env., 1200-1500 m, 14-16.07.2005, S. Murzin leg. - 1 female; Oingchenghou Shan Mts, $70 \mathrm{~km} \mathrm{NW} \mathrm{Chengdu,} 1450 \mathrm{~m}$ 10.07.2004, S. Murzin leg. - 1 female.

Distribution. China (Sichuan — new record; Zhejiang, Jiangxi, Fujian, Taiwan, Guangdong, Hainan, Guangxi, Guizhou), Japan.

\section{Uraecha yunnana Breuning, 1936}

Material. China: S Gansu, S Wenxian, Shangdan, on light, 20.06.2006, Belousov and Kabak leg. - 1 male.

Distribution. China (Gansu - new record; Sichuan, Yunnan).

Menesia sulphurata (Gebler, 1825)

Material. China: Gansu prov., Min Shan Mts., 2100 m, $70 \mathrm{~km} \mathrm{NW}$ Wudu, 1.06.1997, A. Gorodinsky leg. -1 female

Distribution. China (Gansu - new record; Jilin, Beijing,

Hebei, Shanxi, Shandong, Henan, Shaanxi, Ningxia, Hubei, Taiwan, Sichuan), Mongolia, Kazakhstan, Russia, DPR Korea, RO Korea, Japan.

\section{Calloides magnificus (Pic, 1916)}

Material. China: Gansu, Wudu, 21-24.6.2009, E. Kucera leg. -1 female.

Distribution. China (Gansu - new record; Hebei, Shanxi, Shandong, Shaanxi, Sichuan).

Mesosa (Aplocnemia) longipennis Bates, 1873

Material. China: Sicbuan prov., Qingcheng Hou Shan mts., $70 \mathrm{~km}$ NW Chengdu, $1500 \mathrm{~m}, 25-31.08 .2007$, S. Murzin leg. -1 female.

Distribution. China (Sichuan — new record; Yunnan).

Aethalodes verrucosus verrucosus Gahan, 1888

Material. China: Hai Nan, Mt. Wu-zhi-shan, 219.08.2000 - 1 female.

Distribution. China (Hainan - new record; Shaanxi, Zhejiang, Hubei, Jiangxi, Hunan, Fujian, Guangdong, Guangxi, Sichuan, Guizhou), Vietnam.

Paramenesia subcarinata Gressitt, 1951

Material. China: Shaanxi prov., Haozhenzi env., 13501500 m, 14-24.06.1999, S. Murzin - 1 male.

Distribution. China (Shaanxi - new record; Hubei, Guangdong).

\section{Exocentrus theresae Pic, 1939}

Material. China: Sicbuan, WSW Lixian, $5.9 \mathrm{~km} \mathrm{SW}$ Shangzhai, $3025 \mathrm{~m}, 31^{\circ} 20^{\prime} 54^{\prime \prime}$ N, 10250'26" E, 15.06.2016, I. Kabak, G. Davidian leg. -1 male.

Distribution. China (Sichuan — new record; Beijing).
Cleomenes longipennis longipennis Gressitt, 1951

Material. China: Gansu, $70 \mathrm{~km}$ W Wudu, 10.06.1997, A. Shamaev leg. - 1 male (collection of M. Danilevsky, Moscow, Russia).

Distribution. China (Gansu - new records; Shaanxi, Hubei, Taiwan, Sichuan, Yunnan).

Cleomenes semiargentens Gressitt, 1945

Material. China: Shaanxi prov., Zhouzhi env., Taibaishan Nat. park, 1350 m, 30.05.1999, M. Murzin leg. - 2 females.

Distribution. China (Shaanxi - new record; Sichuan).

Cleomenes giganteus Holzschuh, 1995

Material. China: Sichuan prov., valley $5 \mathrm{~km} \mathrm{~N}$ Wenchuan, 2000 m, 28-30.06.2001, S.Murzin leg. - 1 female.

Distribution. China (Sichuan - new record; Yunnan).

Parechthistatus chinensis Breuning, 1942

Material. China: S Gansu prov., Tochizi, $2400 \mathrm{~m}$, 339'21" N 1054'48" E, 21-24.05.1997, S. Murzin leg. 1 male, 1 female; Win Shan Mts., $2100 \mathrm{~m}, 70 \mathrm{~km} \mathrm{NW}$ from Wudu, 1.06.1997, A. Gorodinski leg. - 1 female.

Distribution. China (Gansu - new record; Henan, Shaanxi).

Chloridolum (Leontium) tenuipes (Fairmaire, 1889)

Material. China: Sicbuan, Luojishan mts., $60 \mathrm{~km} \mathrm{~S}$ Xichang, 25.07.1998 - 1 male.

Distribution. China (Sichuan - new record; Guizhou, Yunnan).

Rhodopina albomarmorata Breuning, 1958

Material. China: Sichuan prov., $53 \mathrm{~km} \mathrm{NW} \mathrm{Lixian,}$ 3300-3500 m, 07.2001, S. Murzin leg. - 1 male.

Distribution. China (Sichuan — new record), India: Sikkim and Darjeeling district, Nepal.

Rhodopina pedongensis Breuning, 1969

Material.China: Yunnan prov., $54 \mathrm{~km} \mathrm{E} \mathrm{Tengchong,}$ $2150 \mathrm{~m}, 4-9.09 .2004$, S. Murzin leg. - 1 female.

Distribution. China (Yunnan - new record), India: Sikkim and Darjeeling district.

\section{Annamanum rondoni Breuning, 1962}

Material. S. China: Yunnan prov., Xiquanbanna, Guanping env., $60 \mathrm{~km} \mathrm{~N}$ Jinghong, $1000 \mathrm{~m}, 28-30 . I V .2003$, S. Murzin leg. -1 male.

Distribution. China (Yunnan - new record).

\section{References}

Lin M.-Y. [Meiying] and Yang X.-K. [Xingke] 2019. Catalogue of Chinese Coleoptera volume 9. Chrysomeloidea: Vesperidae, Disteniidae, Cerambycidae. Beijing: Science Press: i-xii, $575 \mathrm{pp}$. 